\title{
Further SAR on the (phenylsulfonyl)piperazine scaffold as inhibitors of the Aedes aegypti Kir1 (AeKir) channel and larvicides
}

Christopher D. Aretz ${ }^{a}$, Sujay V. Kharade ${ }^{b}$, Keagan $_{\text {Chronister }}{ }^{b}$, Renata Rusconi Trigueros ${ }^{c}$, Erick J. Martinez Rodriguez ${ }^{c}$, Peter M. Piermarini ${ }^{c}$, Jerod S. Denton ${ }^{b}$, and Corey R. Hopkins ${ }^{a, *}$

${ }^{a}$ Department of Pharmaceutical Sciences, College of Pharmacy, University of Nebraska Medical Center, Omaha, NE 68198, USA

${ }^{b}$ Department of Anesthesiology, Vanderbilt University Medical Center, Nashville, TN 37232, USA

${ }^{c}$ Department of Entomology, Ohio State University, Wooster, OH 44691, USA

\begin{abstract}
.
Zika virus (ZIKV), dengue fever (DENV) and chikungunya (CHIKV) are arboviruses that are spread to humans from the bite of an infected adult female Aedes aegypti mosquito. As there are no effective vaccines or therapeutics for these diseases, the primary strategy for controlling the spread of these viruses is to prevent the mosquito from biting humans through the use of insecticides. Unfortunately, the commonly used classes of insecticides have seen a significant increase in resistance, thus complicating control efforts. Inhibiting the renal inward rectifier potassium (Kir) channel of the mosquito vector Aedes aegypti has been shown to be a promising target for the development of novel mosquitocides. We have shown that Kirl channels play key roles in mosquito diuresis, hemolymph potassium homeostasis, flight, and reproduction. Previous work from our laboratories identified a novel (phenylsulfonyl)piperazine scaffold as potent $\mathrm{AeKir}$ channel inhibitors with activity against both adult and larval mosquitoes. Herein, we report further SAR work around this scaffold and have identified additional compounds with improved in vitro potency and mosquito larvae toxicity.
\end{abstract}

Keywords. Kir channels, Aedes aegypti, Zika, Dengue, vector-borne diseases, larvae toxicity

\section{Introduction.}

Zika virus (ZIKV) is an arbovirus originating in Africa and Asia that was introduced to South America in 2015 and has quickly expanded throughout the Americas, and is responsible for the high number of reported cases of microcephaly and Guillain-Barré syndrome. ${ }^{1}$ In 2016 , the World Health Organization declared the emerging Zika situation as a "public health emergency of international concern". The virus is primarily transmitted by mosquitoes of the genus Aedes, but the urban-dwelling, anthropophilic Aedes aegypti is suspected as the most important species contributing to the transmission of ZIKV. In addition to ZIKV, Ae. aegypti is the primary vector of the arboviruses that cause chikungunya (CHIKV) and dengue fever (DENV) in humans, which are important diseases that are emerging and reemerging around the globe. Hundreds of millions of people are infected with DENV each year causing hundreds of thousands of hospitalizations and tens of thousands of deaths. ${ }^{2,3}$ Furthermore, there exists substantial economic burdens from DENV with estimated costs of \$2.1 billion in the Americas ${ }^{4}$ and nearly $\$ 1$ billion in Southeast 
Asia. ${ }^{5}$ CHIKV was originally confined to Africa and Southeast Asia, but within the last decade has spread to Europe and further spread to the Americas, where over 400,000 cases and autochthonous transmission have already been reported. ${ }^{6,7}$

There are no effective vaccines and therapeutics available for these viruses and thus, the primary strategy for controlling these diseases is to prevent the mosquito vectors from biting humans, typically by the use of insecticides and/or insecticide-treated materials. ${ }^{8-10}$ However, the progression of resistance in mosquitoes to commonly used classes of insecticides that target the nervous system (e.g., pyrethroids) has severely complicated control efforts. ${ }^{10}$ In addition, as these commonly used insecticides are typically broad-spectrum (i.e., not mosquito selective) their use has been implicated in the decline of pollinators in natural and managed ecosystems (e.g., the honeybee Apis mellifera). ${ }^{11}$ Thus, to overcome the challenge of insecticide resistance in mosquitoes, new control agents with novel modes of action and improved specificity for mosquitoes are needed.

We have previously shown that an inwardly rectifying potassium (Kir1) channel is a promising target for the development of mosquitocides with novel modes of action. Kir1 is expressed in the mosquito Malpighian tubules and ovaries and plays a critical role in renal excretory capacity, hemolymph $\mathrm{K}^{+}$homeostasis, flight and reproduction. We have published on a variety of scaffolds showing toxicity to adult female mosquitoes. ${ }^{12-15}$ More recently, we discovered a new (phenylsulfonyl)piperazine scaffold with superior in vitro potency against AeKir in both a Thallium-flux high-throughput assay as well as in patch clamp assays (Figure 1). ${ }^{16}$ Herein we report further efforts on the structure-activity relationships around this scaffold and their effects on toxicity against mosquito larvae.
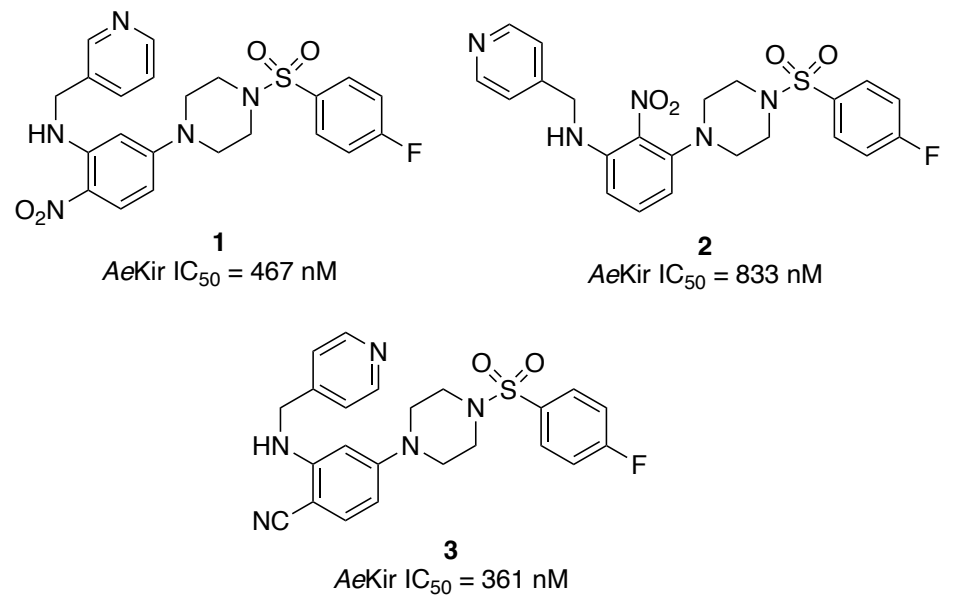

Figure 1. Previously reported (phenylsulfonyl)piperazine inhibitors of AeKir.

\section{Synthesis of Target Compounds.}

The synthetic scheme for the synthesis of the 4-nitrophenylpiperazine analogs has been previously reported and is shown in Scheme $1 .{ }^{16}$ First, piperazine, 4, is reacted with the sulfonyl chloride, $\mathbf{5}$, to yield the key intermediate, $\mathbf{6}$, in quantitative yield. Next, $\mathbf{6}$ and 4-bromo-2-fluoronitrobenzene, 7 , were coupled via palladium catalysis $\left(\mathrm{Pd}(\mathrm{OAc})_{2},( \pm)\right.$-BINAP) to yield the 
penultimate intermediate, $\mathbf{8}$, in good yield (68\%). The final targets were completed by the addition of the appropriate amine to $\mathbf{8}$ under basic conditions to yield compounds $\mathbf{9 a - u}$, in varying yields (4-68\%). The additional synthetic targets outlined in Table 1 procedures are compiled in the Supplemental Information. ${ }^{16}$

Scheme 1. General synthetic scheme for analogs.
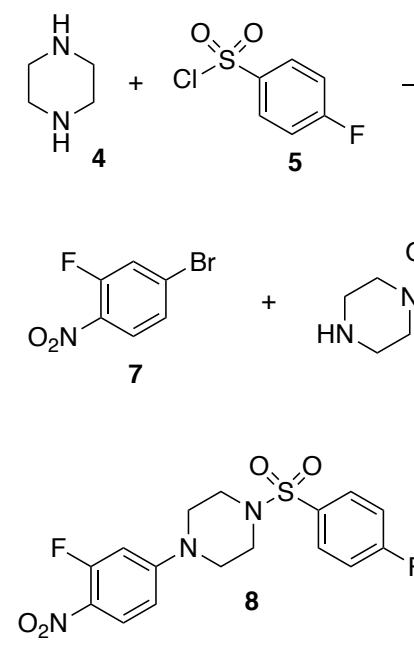

(a)
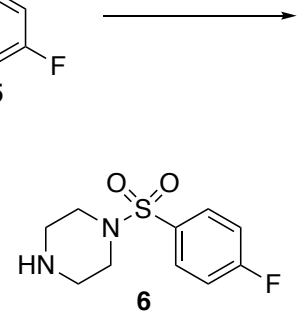

(c)

Reagents and conditions: (a) $\mathrm{CH}_{2} \mathrm{Cl}_{2}, 0^{\circ} \mathrm{C}$, quant.; (b) $\mathrm{Pd}(\mathrm{OAc})_{2}$, ( \pm )-BINAP, $\mathrm{K}_{2} \mathrm{CO}_{3}$, dioxane, reflux, $16 \mathrm{~h} ; 68 \%$; (c) $\mathrm{R}^{-} \mathrm{NH}_{2}, \mathrm{Et}_{3} \mathrm{~N}$, DMSO, $60{ }^{\circ} \mathrm{C}, 16 \mathrm{~h}, 4-68 \%$

The SAR efforts started with the 2-furylmethyl derivative (9a) which lost 3-fold in potency compared to the previous compound, $\mathbf{1},\left(\mathbf{9 a}, \mathrm{IC}_{50}=1.29 \mu \mathrm{M}\right.$ vs. $\left.\mathbf{1}, \mathrm{IC}_{50}=0.467 \mu \mathrm{M}\right)$. Moving from the 2-furyl to the 3 -furyl regained some activity $\left(9 \mathrm{~b}, \mathrm{IC}_{50}=0.713 \mu \mathrm{M}\right)$ against AeKir. Addition of a methyl group to the benzylic position led to a further erosion of activity $\left(9 \mathrm{c}, \mathrm{IC}_{50}=1.65 \mu \mathrm{M}\right)$. Interestingly, addition of a methyl to the 3-position of the furan $\left(9 \mathbf{d}, \mathrm{IC}_{50}=0.546 \mu \mathrm{M}\right)$ was a productive change to the molecule, bringing the activity back in line with the 3-pyridine derivative 1; however, moving the methyl to the 5-position led to a loss of activity $\left(9 \mathrm{e}, \mathrm{IC}_{50}=4.65 \mu \mathrm{M}\right)$. Moving to the isoxazole led to an interesting and surprising result as the 5-methylisoxazole (9f, $\mathrm{IC}_{50}=0.736 \mu \mathrm{M}$ ) regained activity, even though the methyl group is in a similar orientation as the 5-methylfuran, 9e. Further improvements in potency were realized with the 5-methyl-1,3,4oxadiazole moiety, 9g $\left(\mathrm{IC}_{50}=0.441 \mu \mathrm{M}\right)$. Similar activity trends were seen when moving to the thiazole analogs $(\mathbf{9 h}-\mathbf{j})$ as were seen with the furan analogs. The 2-thiazole $\left(\mathbf{9 h}, \mathrm{IC}_{50}=0.496 \mu \mathrm{M}\right)$ retained activity; however, when the benzylic position was methylated, a 3-fold loss in potency was seen $\left(9 \mathrm{i}, \mathrm{IC}_{50}=1.50 \mu \mathrm{M}\right)$. The 2-methyl-5-thiazole regained 2-fold potency (similar to what was seen in the isoxazole analogs) $\left(9 \mathbf{j}, \mathrm{IC}_{50}=0.820 \mu \mathrm{M}\right)$, potentially giving some information regarding the placement of the heteroatoms in the 5-membered ring system.

The next set of analogs we analyzed were the 2-oxazole and 5-oxazole ring systems (9k-o), which provided our most potent compounds to date. The 2-oxazole analog, $9 \mathbf{k}\left(\mathrm{IC}_{50}=0.177 \mu \mathrm{M}\right)$, was 3-fold more potent than the corresponding 2-thiazole, $9 \mathbf{h}\left(\mathrm{IC}_{50}=0.496 \mu \mathrm{M}\right)$ and 7-fold more active than the 2-furan, 9a $\left(\mathrm{IC}_{50}=1.29 \mu \mathrm{M}\right)$. Addition of a methyl substituent to the benzylic position, again, led to a loss of potency $\left(91, \mathrm{IC}_{50}=0.905 \mu \mathrm{M}\right)$. The regioisomeric 5-oxazole 
compound was the most potent compound from this scaffold that we have identified $\left(9 \mathbf{m}, \mathrm{IC}_{50}=\right.$ $0.108 \mu \mathrm{M})$. Addition of a methyl group to the oxazole led to potent compounds; however, the 2methyl-5-oxazole lost considerable potency versus the 5-methyl-2-oxazole $\left(\mathbf{9 o}, \mathrm{IC}_{50}=0.609 \mu \mathrm{M}\right.$ vs. 9n, $\left.\mathrm{IC}_{50}=0.147 \mu \mathrm{M}\right)$. The potency was retained moving from the oxazole to the 1,2,4oxadiazole $\left(9 \mathrm{p}, \mathrm{IC}_{50}=0.190 \mu \mathrm{M}\right)$ or the 5-methyl-1,2,4-oxadiazole $\left(\mathbf{9 q}, \mathrm{IC}_{50}=0.359 \mu \mathrm{M}\right)$ albeit with a slight loss of activity for the 5-methyl derivative. Interestingly, the 3-isoxazole analog lost all activity $\left(9 r, \mathrm{IC}_{50}=30.4 \mu \mathrm{M}\right)$. Lastly, the 5-methyl-1,3,4-thiadiazole analog retained potency $\left(9 \mathrm{~s}, \mathrm{IC}_{50}=0.381 \mu \mathrm{M}\right)$, which is 2-fold more potent than the similarly substituted 2-methyl-5thiazole, $9 \mathbf{j}$. Lastly, extending the ring system in this area to a benzofuran or benzoisoxaole led to dramatic results. The 2-benzofuran, $9 \mathbf{t}$, retained activity compared to the 2-benzisoxazole, $9 \mathbf{u}$, which lost significant potency ( $\mathrm{IC}_{50}=0.381 \mu \mathrm{M}$ vs. $6.12 \mu \mathrm{M}$, respectively).

Moving from the nitrobenzene core to the benzonitrile core led to a few interesting observations. First, the unsubstituted 2-amino analog, 12a, was the most potent compound in this series $\left(\mathrm{IC}_{50}=0.237 \mu \mathrm{M}\right)$. Further substitution of the aniline with the oxazole moieties led to potent compounds $\left(\mathbf{1 2 b}-\mathbf{d}, \mathrm{IC}_{50}=0.404-1.56 \mu \mathrm{M}\right)$; however, the benzonitrile compounds were less potent than their nitrobenzene counterparts. Additional analogs were synthesize moving the nitro or cyano group from para to the piperazine to ortho was an unproductive change as all compounds were significantly less potent $\left(\mathbf{1 5 a}-\mathbf{d}, \mathbf{2 1 a}, \mathrm{IC}_{50}>9.8 \mu \mathrm{M}\right)$, which is in contrast to the pyridine analog, 2. Lastly, the simplified 3-fluoro-4-nitro derivative, 8 , had reduced activity $\left(\mathrm{IC}_{50}=2.43\right.$ $\mu \mathrm{M})$.

Table 1. SAR of key nitro- and cyanobenzene derivatives.

\begin{tabular}{|c|c|c|c|}
\hline Cmpd & Structure & $\begin{array}{c}A e \text { Kir } \\
\text { Thallium Flux } \\
\text { IC }_{50}(\mu \mathrm{M})^{a}\end{array}$ & $\begin{array}{c}\% \\
\text { Inhibition }^{a}\end{array}$ \\
\hline $9 a$ & & $1.29 \pm 0.10$ & 96 \\
\hline $9 b$ & & $0.713 \pm 0.084$ & 110 \\
\hline $9 \mathrm{c}$ & & $1.65 \pm 0.13$ & 95 \\
\hline 9d & & $0.546 \pm 0.045$ & 92 \\
\hline $9 e$ & & $4.51 \pm 0.451$ & 85 \\
\hline
\end{tabular}




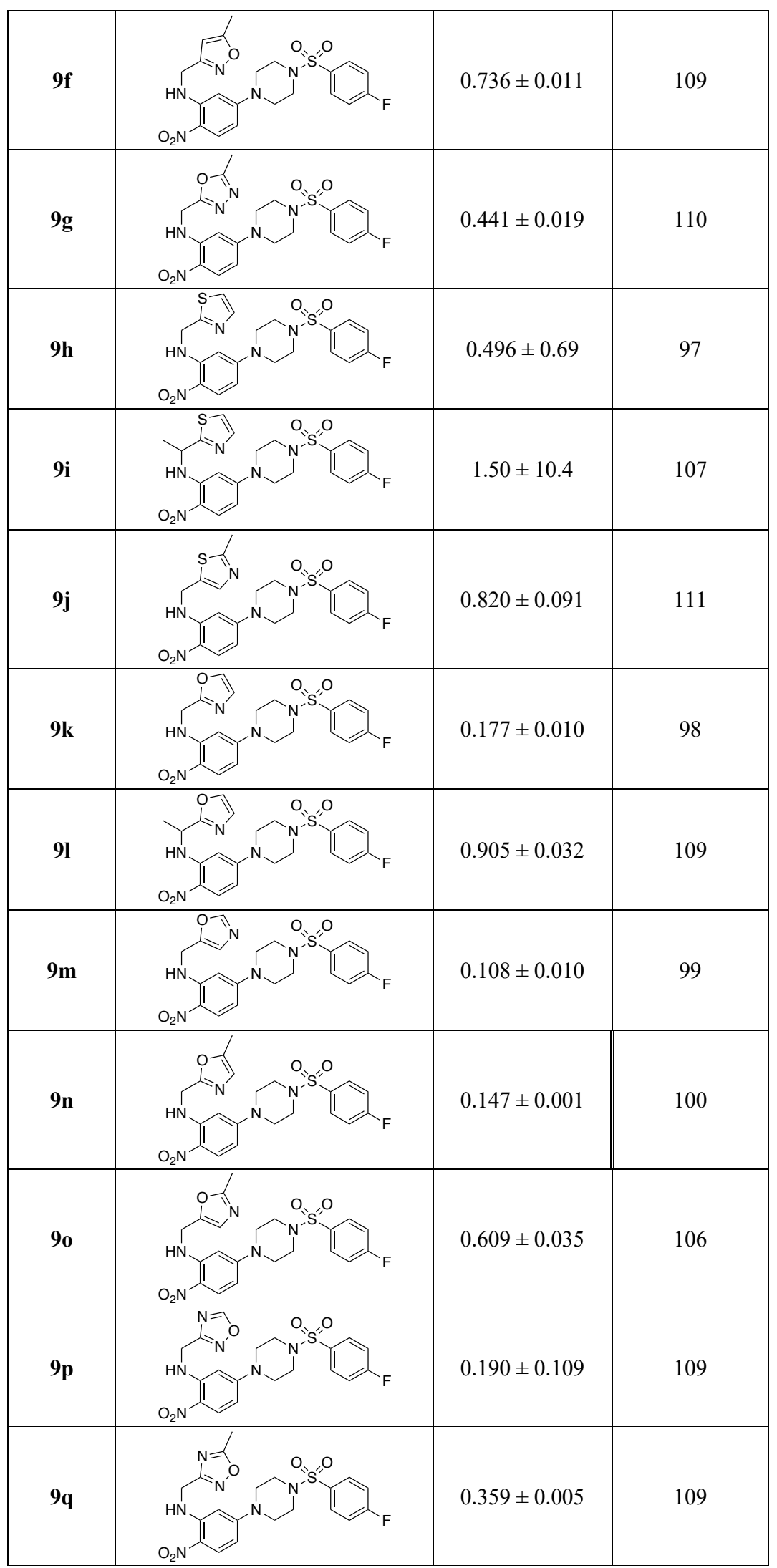




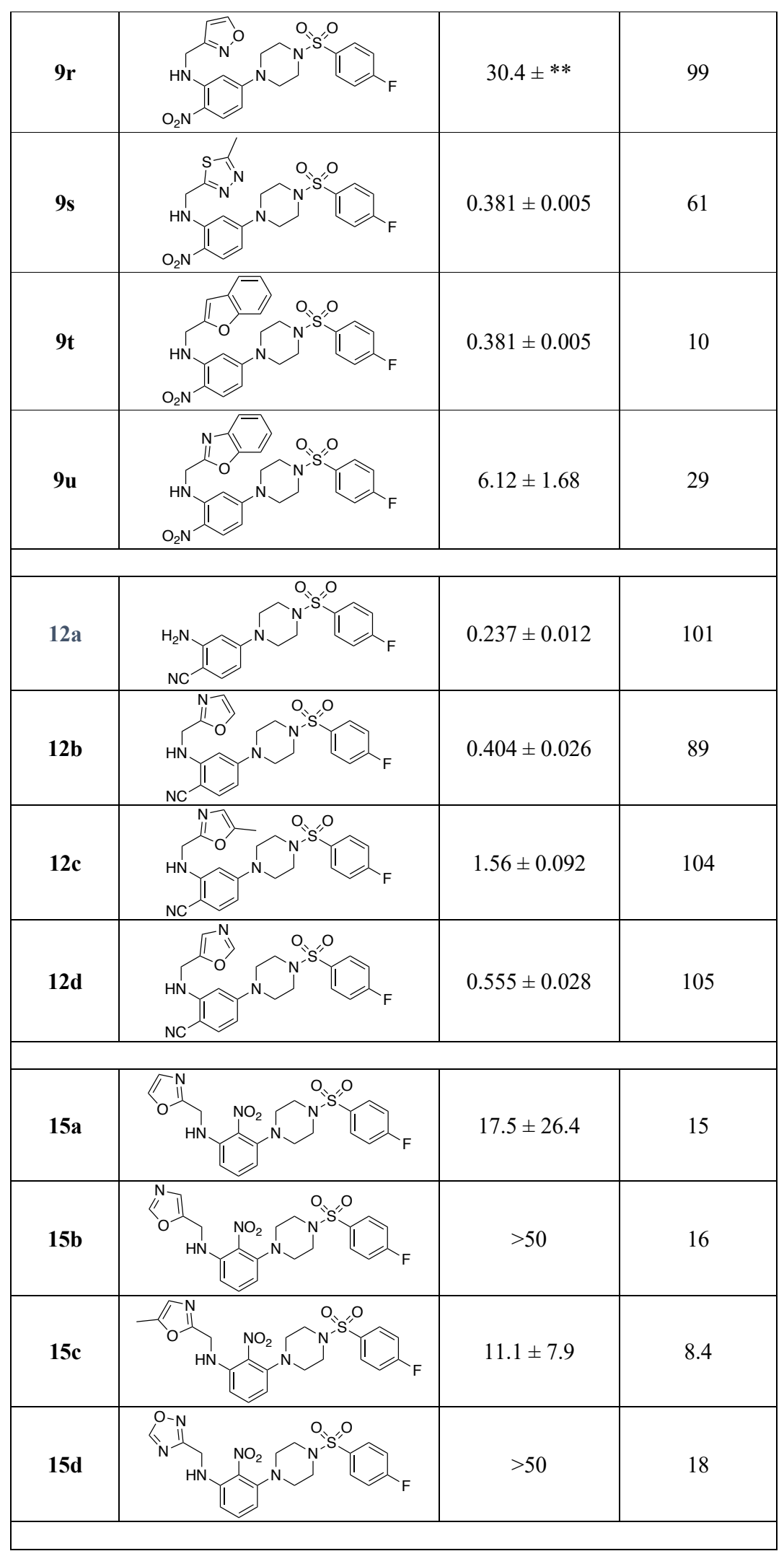




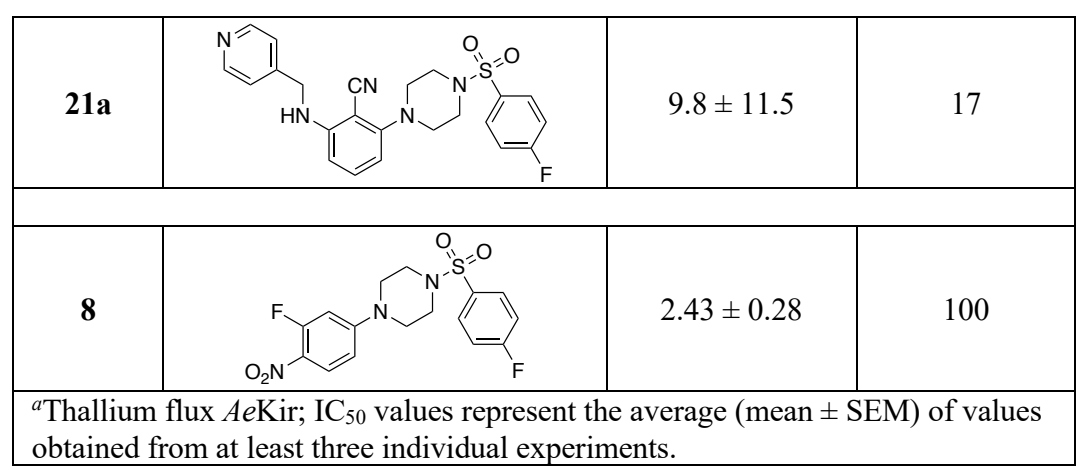

Having identified a number of potent $A e K i r$ inhibitors, we next screened a selection of these compounds for larvae toxicity at $100 \mu \mathrm{M}$ and compared their efficacies to previously reported VU854. ${ }^{16}$ As with other compounds, the new analogs had limited activity at $24 \mathrm{~h}(<25 \%$, data not shown); however, many of the new analogs had significant mortality at $48 \mathrm{~h}$ (Figure 2). Compounds 8 (100\%), 9p (99\%) and 12d (98\%) showed significantly greater mortality at $48 \mathrm{~h}$ compared to VU854 (shown in red, Figure 2). ${ }^{16}$ A number of other compounds $(\mathbf{9 n}, \mathbf{9 q}, \mathbf{9 s}, \mathbf{1 2 a}$ and 12b) were similarly efficacious as VU854 (shown in grey, Figure 2), whereas others were less efficacious than VU854 (shown in blue, Figure 2). These results are intriguing as compounds that are active in the larval toxicity assay are not the most potent in vitro (e.g., 8) which raises questions to whether a compound such as $\mathbf{8}$ is more permeable versus other, more potent, compounds (e.g., 9k). In addition, $\mathbf{8}$ is devoid of the amino substituent which, previously, has been important for activity (e.g., hydrogen substituents were inactive). Further work concentrated on determining the effects of in vitro potency on larval toxicity are underway and will be reported in due course.

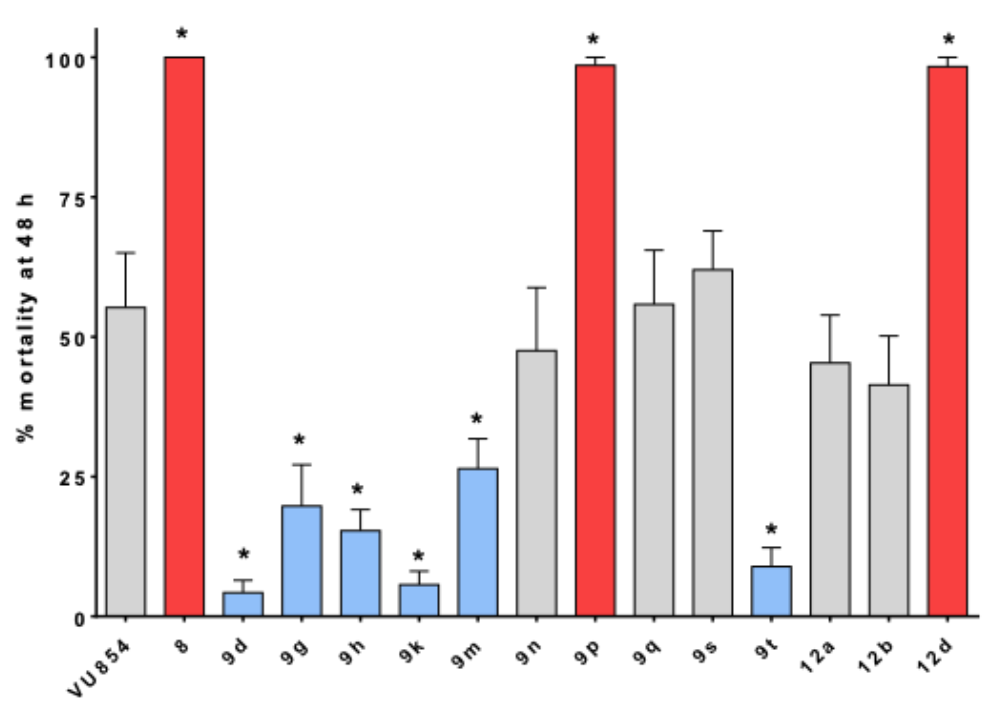

\begin{tabular}{|c|c|}
\hline Cmpd & Larvae toxicity at $\mathbf{4 8}$ h \\
\hline VU854 & $55.3 \pm 9.8$ \\
\hline $\mathbf{8}$ & $100 \pm 0$ \\
\hline 9d & $4.3 \pm 2.2$ \\
\hline 9g & $19.8 \pm 7.4$ \\
\hline 9h & $15.3 \pm 3.8$ \\
\hline 9k & $5.7 \pm 2.4$ \\
\hline 9m & $26.4 \pm 5.3$ \\
\hline 9n & $47.5 \pm 11.3$ \\
\hline 9p & $98.6 \pm 1.4$ \\
\hline 9q & $55.8 \pm 9.7$ \\
\hline 9s & $62.0 \pm 7.0$ \\
\hline 9t & $8.9 \pm 3.4$ \\
\hline 12a & $45.3 \pm 8.6$ \\
\hline 12b & $41.4 \pm 8.8$ \\
\hline 12d & $98.3 \pm 1.7$ \\
\hline
\end{tabular}

Figure 2. $48 \mathrm{~h}$ mortality of first instar (Liverpool, LVP) Ae. aegypti after addition of small molecules $(100 \mu \mathrm{M})$ to the rearing water. Values are means \pm SEM based on at least 12 replicates. 
Lastly, we evaluated the top three compounds $(\mathbf{8}, \mathbf{9 p}, \mathbf{1 2 d})$ in a concentration response curve (CRC) experiment against both the LVP (pyrethroid-susceptible) and the Puerto Rico (PR) strain (pyrethroid-resistant) to assess their toxicity (Figure 3). As can be seen in Figure 3A, within $48 \mathrm{~h}$, VU854 is equipotent against both strains with maximal mortality reaching $\sim 50 \%$ at $100 \mu \mathrm{M}$. Both $\mathbf{8}$ and 12d, reached $100 \%$ mortality within $48 \mathrm{~h}$ at $100 \mu \mathrm{M}$, with $\mathbf{1 2 d}$ showing no differences between the strains, whereas 8 was $\sim 2$-times more potent against the LVP strain. Disappointingly, in the CRC experiments, 9p did not recapitulate the high efficacy found in the screening experiments (Figure 2) even after repeated testing. Its maximal mortality was limited to $\sim 50 \%$ within $48 \mathrm{~h}$ at $100 \mu \mathrm{M}$. However, 9p consistently produced a unique pathology in the larvae (Figure 3E) wherein an accumulation of a contignuous trail of excrement (magenta arrows) that began in the midgut and extended continuously beyond the rectum, indicative of constipation or excretory failure. We have not seen this result with any other compound tested. We are currently evaluating the methods and will report any differences in due course.

A
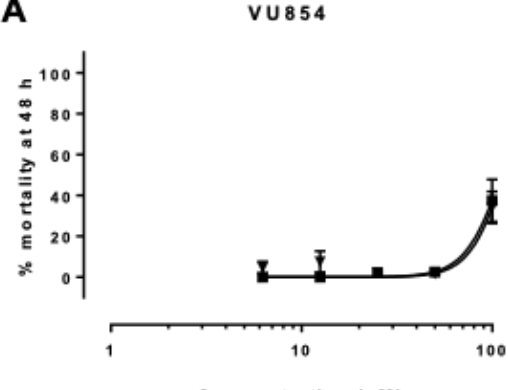

Concentration $(\mu M)$

C

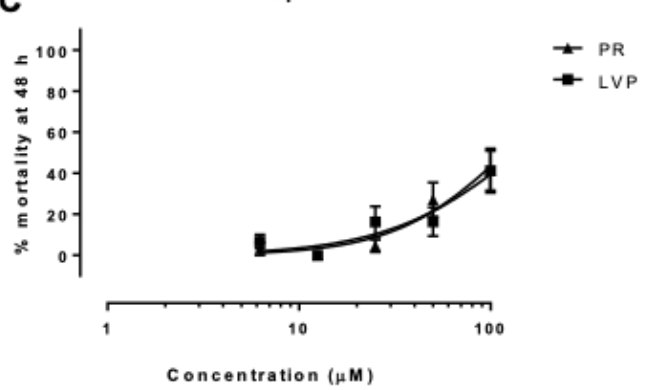

E
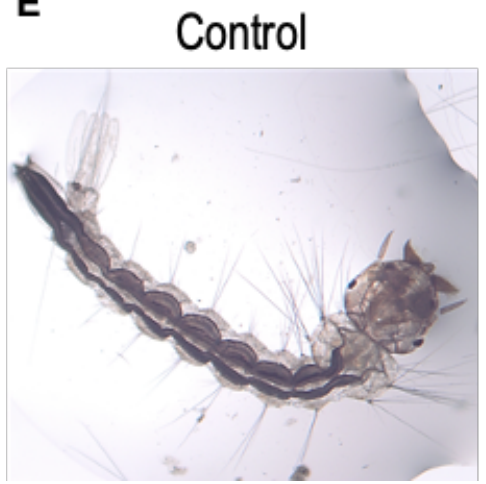

$\rightarrow P R$

- LVP

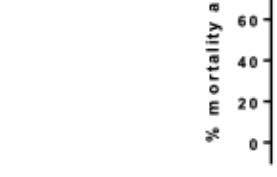

B 8

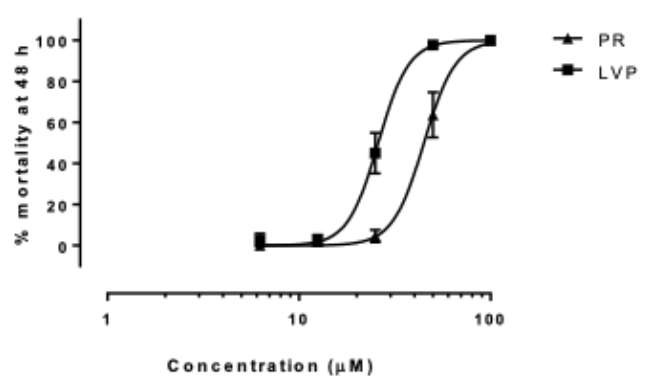

D

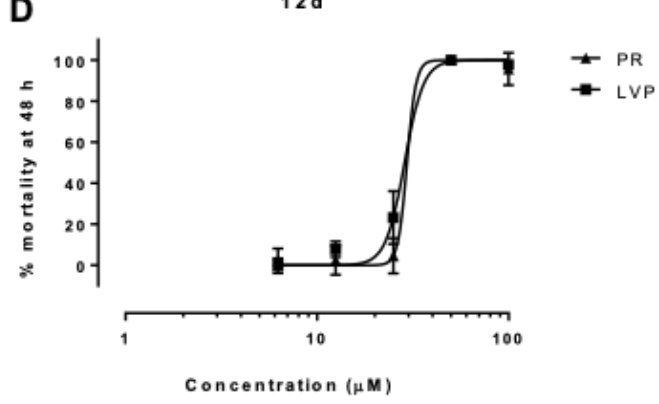

$9 \mathrm{p}(48 \mathrm{~h})$

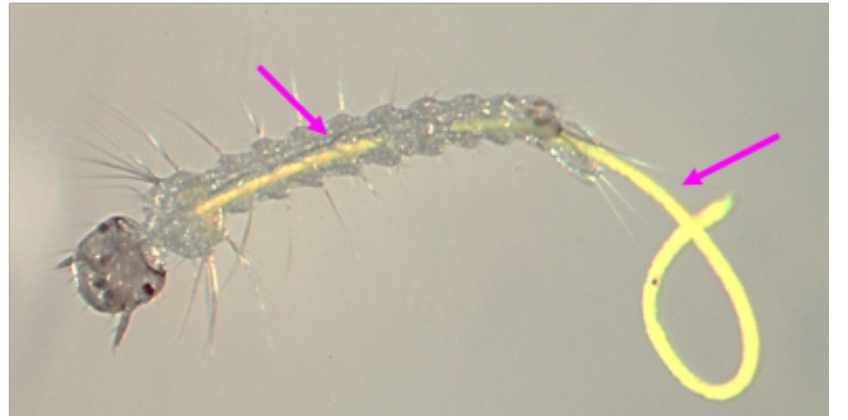


Figure 3. (A-D) $48 \mathrm{~h}$ mortality of first instar (LVP or PR) Ae. aegypti after addition of small molecules to the rearing water at various concentrations. (E) Excretory failure (magenta arrows) obseved in larvae treated with $\mathbf{9 p}$.

\section{Conclusions.}

We have reported on further SAR evaluation of our previously reported (phenylsulfonyl)piperazine scaffold, by expanding on the heterocyclic moieties explored around the phenyl ring. We have discovered a number of compounds that are more potent in the Thalliumflux in vitro assay $(<200 \mathrm{nM})$. SAR results indicate that the oxazole and oxadiazole heterocycles were the optimal group, and cyano for nitro substitution was also tolerated. In addition, $\mathbf{8 , 9 p}$ and 12d show excellent efficacy against Aedes aegypti larvae in a $48 \mathrm{~h}$ first instar toxicity assay and $\mathbf{8}$ and 12d were potent in a CRC against both the LVP and PR strains of Ae. aegypti, suggesting these compounds could be used to combat resistance seen in pyrethroids. Compound $\mathbf{8}$ was also unique as it did not contain the heterocyclic substituted amino group that has been seen to be optimal in previous studies. Further optimization and adult lethality studies are on-going and will be reported in due course.

\section{Acknowledgments.}

The authors would like to thank the National Institute of Allergy and Infectious Disease (NIAID) for an R21 award to CRH, JSD and PMP (R21 AI128418).

\section{References.}

1. Fauci, A. S.; Morens, D. M., Zika virus in the Americas - Yet another arbovirus threat. $N$. Engl. J. Med. 2016, 374 (7), 601-604.

2. Bhatt, S.; Gething, P. W.; Brady, O. J.; Messina, J. P.; Farlow, A. W.; Moyes, C. L.; Drake, J. M.; Brownstein, J. S.; Hoen, A. G.; Sankoh, O.; Myers, M. F.; George, D. B.; Jaenisch, T.; Wint, G. R. W.; Simmons, C. P.; Scott, T. W.; Farrar, J. J.; Hay, S. I., The global distribution and burden of dengue. Nature 2013, 496, 504-507.

3. WHO, Global strategy for dengue prevention and control. Geneva: World Health Organization. 2012, 43 p.

4. Shepard, D. S.; Coudeville, L.; Halasa, Y. A.; Zambrano, B.; Dayan, G. H., Economic impact of Dengue illness in the Americas. Am. J. Trop. Med. Hyg. 2011, 84 (2), 200-207.

5. Shepard, D. S.; Undurraga, E. A.; Halasa, Y. A., Economic and disease burden of Dengue in southeast Asia. PLoS Negl. Trop. Dis. 2013, 7 (2), e2055.

6. Morrison, T. E., Reemergence of Chikungunya virus. J. Virol. 2014, 88 (20), 11644-11647.

7. Weaver, S. C., Arrival of Chikungunya virus in the new world: prospects for spread and impact on public health. PLoS Negl. Trop. Dis. 2014, 8 (6), e2921.

8. Vontas, J.; Kioulos, E.; Pavlidi, N.; Morou, E.; della Torre, A.; Ranson, H., Insecticide resistance in the major Dengue vectors Aedes albopictus and Aedes aegypti. Pest. Biochem. Physiol. 2012, 104, 126-131.

9. Ranson, H.; Burhani, J.; Lumjuan, N.; Black, W. C., IV, Insecticide resistance in dengue vectors. TropIKA.net [online] 2010, 1 (1), 0-0.

10. Shaw, W. R.; Catteruccia, F., Vector biology meets disease control: using basic research to fight vector-borne disesaes. Nat. Microbiol. 2019, 4, 20-34. 
11. Goulson, D.; Nicholls, E.; Botías, C.; Rotheray, E. L., Bee declines driven by combined stress from parasites, pesticides, and lack of flowers. Science 2015, 347 (6229), 1255957.

12. Raphemot, R.; Rouhier, M. F.; Hopkins, C. R.; Gogliotti, R. D.; Lovell, K. M.; Hine, R. M.; Ghosalkar, D.; Longo, A.; Beyenbach, K. W.; Denton, J. S.; Piermarini, P. M., Eliciting renal failure in mosquitoes with a small-molecule inhibitor of inward-rectifying potassium channels. PLoS One 2013, 8 (5), e64905.

13. Raphemot, R.; Rouhier, M. F.; Swale, D. R.; Days, E.; Weaver, C. D.; Lovell, K. M.; Konkel, L. C.; Engers, D. W.; Bollinger, S. F.; Hopkins, C.; Piermarini, P. M.; Denton, J. S., Discovery and characterization of a potent and selective inhibitor of Aedes aegypti inward rectifying potassium channels. PLoS One 2014, 9 (11), e110772.

14. Rouhier, M. F.; Raphemot, R.; Denton, J. S.; Piermarini, P. M., Pharmacological validation of an inward-rectifier potassium (Kir) channel as an insecticide target in the yellow fever mosquito Aedes aegypti. PLoS One 2014, 9 (6), e100700.

15. Swale, D. R.; Engers, D. W.; Bollinger, S. R.; Inocente, E. A.; Days, E.; Kanga, F.; Johnson, R. M.; Yang, L.; Bloomquist, J. R.; Hopkins, C. R.; Piermarini, P. M.; Denton, J. S., An insecticide resistance-breaking mosquitocide targeting inward rectifier potassium channels in vectors of Zika virus and malaria. Sci. Rep. 2016, 6, 36954.

16. Aretz, C. D.; Morwitzer, M. J.; Sanford, A. G.; Hogan, A. M.; Portillo, M. V.; Kharade, S. V.; Kramer, M.; McCarthey, J. B.; Trigueros, R. R.; Piermarini, P. M.; Denton, J. S.; Hopkins, C. R., Discovery and characterization of 2-nitro-5-(4-(phenylsulfonyl)piperazin-1yl)- $N$-(pyridin-4-ylmethyl)anilines as novel inhibitors of the Aedes aegypti Kir1 (AeKir1) channel. ACS Infect. Dis. 2019, 5, 917-931. 